\title{
Fourier-series expansion of the dark-energy equation of state
}

\author{
David Tamayo* \\ Departamento de Física, Centro de Investigación y de Estudios Avanzados del IPN, A.P. 14-740, 07000 México D.F., México.
}

\author{
J. Alberto Vazquez ${ }^{\dagger}$ \\ Instituto de Ciencias Fisicas, Universidad Nacional Autonoma de Mexico, \\ Apdo. Postal 48-3, 62251 Cuernavaca, Morelos, Mexico
}

\begin{abstract}
The dark-energy component of the Universe still remains a mystery; however, several papers based on observational data have shown that its equation of state may have an oscillatory behaviour. In this paper, we provide a general description for the dark-energy equation of state $w(z)$ in the form of a Fourier series. This description generalizes some previous dynamical dark-energy models and is in agreement with the $w(z)$ reconstructions. We make use of a modified version of a simple and fast Markov chain Monte Carlo code to constrain the model parameters. For the analysis we use data from supernovae type Ia, baryon acoustic oscillations, $H(z)$ measurements and cosmic microwave background. We provide a comparison of the proposed model with $\Lambda$ CDM, $w C D M$ and the standard Taylor approximation. The Fourier-series expansion of $w(z)$ is preferred from $\Lambda$ CDM at more than the $3 \sigma$ significance level based on the improvement in the fit alone. We use the Akaike criterion to perform the model comparison and find that, even though there are extra parameters, there is a slight preference for the Fourier series compared with the $\Lambda$ CDM model. The preferred shape of $w(z)$ found here puts in jeopardy the single scalar field models, as they cannot reproduce the crossing of the phantom divide line $w=1$.
\end{abstract}

*dtamayo@fis.cinvestav.mx

† javazquez@icf.unam.mx 


\section{INTRODUCTION}

The dark energy is still an unknown negative pressure cosmic component for which the simplest case is given in terms of a perfect fluid with an equation of state (EoS) $p=w \rho$ with $w=-1$, this particular model is commonly named as the cosmological constant $\Lambda$ and it is a key piece of the standard cosmological model: the $\Lambda$ CDM model. Even though the standard cosmological model fits well with most of the current astronomical observations, there exist important tensions among different recent data sets. For instance, the value of $H_{0}$ measured from CMB data by the Planck Collaboration [1] is $3.4 \sigma$ lower than the local value reported by Riess et al. [2]. The matter density fraction consistent with the Lyman- $\alpha$ forest measurement of the baryon acoustic oscillations (BAO) is smaller than the one preferred by CMB measurements [3]. On the other hand, based on the most minimal a priori assumptions, model independent reconstructions of the evolution of the dark energy EoS parameter exhibit a dynamical behaviour of $w(z)$ [4-7], putting in tension the $\Lambda$ CDM model amongst several models for which $w=$ constant. From the theoretical point of view, the standard cosmological model also carries out several important theoretical problems such as the absence of physical grounds to justify the cosmological constant, the coincidence problem and fine tuning, see [8-10]. In order to get around these issues, there have been plenty of proposals to describe the general behaviour of this dark component, i.e. scalar fields (quintessence, K-essence, phantom, quintom, non-minimally coupled scalar fields, etc.) [11], modified gravity [12], interacting dark energy [13] and divergence free parameterizations [14], among many others $[15]$.

Dark energy parameterizations of the EoS parameter are a set of phenomenological models that consist of assuming that the dark energy behaves as a perfect fluid with a dynamical equation of state, that is $p=w(z) \rho$, without any other assumption about the origin of this behaviour from fundamental physics. The main goal of this approach is to model the time evolution of $w(z(t))$ from observational data which can give important insights about the evolution of the dark energy and put a phenomenological basis for its theoretical description. Here we briefly summarise some of these models.

First, one of the most popular time-evolving parameterizations consists of expanding $w$ in a Taylor series around $a=1$ (today): $w(a)=\sum_{i=0}^{N}(1-a)^{i} w_{i}$, where $N$ defines the order of the polynomial expansion and $w_{i}$ are constant values. The trivial case, when $N=0$, is $w(a)=w_{0}$ also known as $w$ CDM model, and clearly for the particular case of $w_{0}=-1$ we return to the well-known cosmological constant. The case $N=1$, i.e. $w(a)=w_{0}+(1-a) w_{1}$ corresponds to the very well-known Chevallier-Polarski-Linder (CPL) model [16, 17], this richer model has been widely studied [18] and can be mapped into another dark energy models like quintessence and barotropic dark energy [19]. A recent study of several cases of wCDM and CPL models can be found in [20], here the authors compare the models by performing different statistical criteria in order to highlight the differences. For higher orders $N=2,3,4$, a nice analysis is performed in [21], the authors by analysing cosmological data (SNIa, CMB, LSS, H(z), BAO) conclude that the concordance cosmological constant model $(w=-1)$ is still safely consistent with these observational data at the $68 \%$ confidence level. However, when adding the high-redshift BAO measurement from the Ly $\alpha$ forest of BOSS DR11 quasars into the calculation there is a significant impact on the reconstruction result, the $w$ prefers values significantly smaller than -1 .

Parameterizations which use power laws and exponential functions with two and three free parameters were analysed by Martins et al. in [22]. Through a standard statistical analysis of cosmological data (SNIa, $H(z))$ they set constraints at the present-day values of the dark energy EoS and in the asymptotic past in these models. They conclude that the dark energy EoS near the present day must be very similar to that of a cosmological constant, and any significant deviations from this behaviour can only occur in the deep matter era.

Another approach consists in phenomenological models using trigonometric functions. A first attempt was proposed by Linder arguing that oscillating dark energy models offer one idea for solving the coincidence problem [23], the ansatz considers an EoS parameter with the form of $w(a)=w_{0}-A \sin (B \ln a)$ where the natural period of the cosmic expansion is given by $H^{-1}=(d \ln a / d t)^{-1}$, so they examine periodicity in units of the $e$-folding scale ln $a$. A further work of Pace et al. [24] studies the imprints on the formation and evolution of cosmic structures in six different variations of this particular dark energy model. Moreover, from a phantom scalar field conformally coupled to gravity model Kurek et al. obtain a parameterization of the type $w(z)=-1+(1+z)^{3}\left[C_{1} \cos (\ln (1+z))+C_{2} \sin (\ln (1+z))\right]$ [25]. In the reference [26] Pan et al. study several oscillating dark energy models: $w_{I}=w_{0}+b[1-\cos (\ln (1+z))]$, $w_{I I}=w_{0}+b \sin (\ln (1+z)), w_{I I I}=w_{0}+b\left[\frac{\sin (1+z)}{1+z}-\sin 1\right]$ and $w_{I V}=w_{0}+b\left[\frac{z}{1+z} \cos (1+z)\right]$, see references therein to see the motivation of the selection of these particular phenomenological models. They perform a confrontation of these dark energy parameterizations with observational data (JLA, SNIa, BAO, CMB, redshift space distortion, weak gravitational lensing, $H(z))$ they find that the best-fit characters of almost all models are bent towards the phantom region; nevertheless, in all of them, the quintessential regime is also allowed within $1 \sigma$ confidence level. Finally, they perform the Bayesian analysis, which shows that the current observational data supports the $\Lambda$ CDM paradigm over this set of oscillating dark energy parameterizations. A complementary study for this set of models is done in [27], where 
the authors compute the statefinder parameters, and at the level of linear cosmological perturbations they compute the growth index as well as the combination parameter $f \sigma_{8}$, obtaining similar conclusions. In Jaime et al. [28] the authors propose a cosine-like parameterization for the dark energy EoS with the form $w=-1+\frac{w_{0}}{1+w_{1} z^{w_{2}}} \cos \left(w_{3}+z\right)$ which can reproduce some successful $f(R)$ gravity models with a precision between [0.5\%-0.8\%] over the numerical solutions, using observational data from BAO, SNIa, and cosmic chronometers they investigate the constraints on the new EoS parameters.

The aforementioned reconstruction of $w(z)$ from observational data shows that the shape of $w(z)$ crosses the phantom divide line (PDL) $w=-1$ several times, having an oscillating behaviour and puts in jeopardy many dark energy models such as $\Lambda \mathrm{CDM}$ and single scalar field models that are unable to cross the PDL. This oscillating $w(z)$ can be modelled with Taylor series of third order or more, but has its limitations; first, the expansion has to be done around a specific point which commonly is around $z=0$ and this implies that far away from the expansion point the approximation may not be accurate; second, the expansion is done with polynomials and to describe an oscillatory function many terms are needed to get a good representation and third, sufficiently far from $z=0$ the polynomial always grows or decreases monotonically.

If we want to model an oscillating $w(z)$, trigonometric functions are a natural choice and therefore in this work we generalise this idea using a general description of $w(z)$ in the form of Fourier series. This approach avoids the Taylor expansion problems mentioned before: the expansion is done over a period and not a point, there are needed less terms of the series to reproduce well the oscillations and the trigonometric functions are bounded. We show that the Fourier series approach fits better to the data than Taylor expansion models.

The present work is organised in the following way: first in section (II) we provide a general description for the model proposed in this work. Then in section (III) the methodology of the data analysis is done. The results and constraints of the proposed models as well as its comparison with different models are shown in (IV). Finally the conclusion and discussion of the results are presented in (V).

\section{THE OSCILLATING DARK ENERGY MODEL}

The general idea of this work is to consider the EoS parameter $w(a)$ as a Fourier series in the interval $1 \geq a \geq a_{m e d}$ (remembering the convention of the value of the scale factor at present time $a\left(t_{0}\right) \equiv 1$ ), a linear adjust in the interval $a_{\text {med }} \geq a \geq a_{\text {ini }}$ and $w=-1$ otherwise. The motivation of selecting these intervals is the following. First, the available data shows a possible oscillating behaviour of $w$ at low redshifts $(0 \leq z \lesssim 2.5)$ [5], thus the first natural approximation is to use trigonometric functions and then in a more general form use the Fourier series. Then, at higher redshifts $(z \gtrsim 3)$ there is not enough evidence about the behaviour of $w$ different from the cosmological constant, i.e. we use $w=-1$. To join these two different behaviours in a continuous way we use a transitional interval in which $w$ is linear. We impose that at the transition points, $a_{m e d}$ and $a_{i n i}$, the value of the functions used by our model is continuous, $w_{F}\left(a_{m e d}\right)=w_{L}\left(a_{m e d}\right)$ and $w_{L}\left(a_{i n i}\right)=w_{C}=-1$, where the subscripts $F, L, C$ correspond to the Fourier, linear and constant parts of $w$. Therefore, the piecewise function of the EoS parameter $w$ in terms of the scale factor is:

$$
w(a)= \begin{cases}-1 & a_{\text {ini }}>a, \\ m\left(a-a_{i n i}\right)+b & a_{\text {med }} \geq a \geq a_{\text {ini }}, \\ \frac{w_{0}}{2}+\sum_{n=1}^{\infty}\left(a_{n} \sin \left[\frac{2 n \pi}{T}\left(a-a_{m e d}\right)\right]+b_{n} \cos \left[\frac{2 n \pi}{T}\left(a-a_{m e d}\right)\right]\right) & 1 \geq a \geq a_{\text {med }} .\end{cases}
$$

In terms of the redshift $a=(z+1)^{-1}$ we have

$$
w(z)= \begin{cases}-1 & z_{\text {ini }}<z, \\ m\left(\frac{1}{z+1}-\frac{1}{z_{i n i}+1}\right)+b & z_{m e d} \leq z \leq z_{\text {ini }}, \\ \frac{w_{0}}{2}+\sum_{n=1}^{\infty}\left(a_{n} \sin \left[\frac{2 n \pi}{T}\left(\frac{1}{z+1}-\frac{1}{z_{\text {med }}+1}\right)\right]+b_{n} \cos \left[\frac{2 n \pi}{T}\left(\frac{1}{z+1}-\frac{1}{z_{\text {med }}+1}\right)\right]\right) & 0 \leq z \leq z_{m e d} .\end{cases}
$$

where $T=1-a_{m e d}$ is the period. The constants of the linear equation, $m$ and $b$, can be calculated from the equation of a line given two points $\left(P_{1}, P_{2}\right)$ :

$$
\begin{aligned}
& P_{1}=\left(a_{\text {med }}, w\left(a_{\text {med }}\right)\right) \equiv\left(a_{\text {med }}, w_{\text {med }}\right)=\left(a_{\text {med }}, \frac{w_{0}}{2}+\sum_{n=1}^{\infty} b_{n}\right), \\
& P_{2}=\left(a_{\text {ini }}, w\left(a_{\text {ini }}\right)\right) \equiv\left(a_{\text {ini }}, w_{\text {ini }}\right)=\left(a_{\text {med }},-1\right)
\end{aligned}
$$


therefore

$$
\begin{aligned}
m & =\frac{w_{m e d}-w_{i n i}}{a_{m e d}-a_{i n i}}=\frac{\frac{w_{0}}{2}+\sum_{n} b_{n}+1}{a_{m e d}-a_{i n i}} \\
b & =w_{i n i}=-1
\end{aligned}
$$

To calculate the dark energy density $\rho_{d e}$ we have to solve the conservation equation

$$
\begin{array}{r}
\dot{\rho}_{d e}+3 H(1+w) \rho_{d e}=0, \\
\Rightarrow \quad \rho_{d e}=A \exp \left[-3 \int(1+w) \frac{d a}{a}\right],
\end{array}
$$

where $A$ is the integration constant. Performing the integral for the different parts of $w(a)$ we have:

$$
\begin{aligned}
\rho_{F}= & A_{F} a^{-3\left(1+\frac{w_{0}}{2}\right)} \exp \left[-3 \sum_{n=1}^{\infty} \operatorname{Ci}(\operatorname{an} \theta)\left[b_{n} \cos \left(a_{m e d} n \theta\right)-a_{n} \sin \left(a_{m e d} n \theta\right)\right]\right] \times \\
& \exp \left[-3 \sum_{n=1}^{\infty} \operatorname{Si}(\operatorname{arn} \theta)\left[a_{n} \cos \left(a_{m e d} n \theta\right)+b_{n} \sin \left(a_{m e d} n \theta\right)\right]\right], \\
\rho_{L}= & A_{L} a^{1+b+3 m a_{i n i}} e^{3 m a}, \\
\rho_{C}= & A_{C} .
\end{aligned}
$$

Where $A_{F}, A_{L}, A_{C}$ are the integration constants for each particular case, $\theta=2 \pi / T$ and, Ci and Si are the cosine integral and sine integral defined as:

$$
\operatorname{Ci}(x)=-\int_{x}^{\infty} \frac{\cos t}{t} d t, \quad \operatorname{Si}(x)=\int_{0}^{x} \frac{\sin t}{t} d t,
$$

which in the interval $1 \geq a \geq a_{\text {med }}$ are well defined. The integration constants are calculated by solving the system: $\rho_{F}\left(a_{m e d}\right)=\rho_{L}\left(a_{m e d}\right), \rho_{L}\left(a_{i n i}\right)=\rho_{C}\left(a_{i n i}\right)$ and to close the system we use $\rho_{F}\left(a\left(t_{0}\right)\right) \equiv \rho_{0}$ (the energy density of the dark energy today). Solving, we obtain the integration constants:

$$
\begin{aligned}
& A_{F}=\rho_{d e}^{(0)} \exp \left[3 \sum_{n=1}^{\infty}\left[\operatorname{Ci}(n \theta) f_{n}+\operatorname{Si}(n \theta) g_{n}\right]\right] \\
& A_{L}=A_{F} a_{m e d}^{-3\left(\frac{w_{0}}{2}-b+a_{i n i} m\right)} \exp \left[3 \sum_{n=1}^{\infty}\left[a_{m e d} m-f_{n} \operatorname{Ci}\left(a_{m e d} n \theta\right)-g_{n} \operatorname{Si}\left(a_{m e d} n \theta\right)\right]\right] \\
& A_{C}=A_{L} a_{i n i}^{-3\left(1+b-a_{i n i} m\right)} e^{-3 a_{i n i} m}
\end{aligned}
$$

where $\rho_{d e}^{(0)}=\rho_{d e}(a=1)$ and we have defined the auxiliary functions

$$
\begin{aligned}
& f_{n}=b_{n} \cos \left(a_{\text {med }} n \theta\right)-a_{n} \sin \left(a_{\text {med }} n \theta\right), \\
& g_{n}=a_{n} \cos \left(a_{\text {med }} n \theta\right)+b_{n} \sin \left(a_{\text {med }} n \theta\right) .
\end{aligned}
$$

Given the energy density of the dark energy, we can calculate the Hubble function for a cosmological model of a universe filled with matter (baryonic and dark matter), radiation (photons, neutrinos, etc.) and dark energy:

$$
H=H_{0}\left(\Omega_{m}^{(0)} a^{-3}+\Omega_{r}^{(0)} a^{-4}+\Omega_{d e}^{(0)} \rho_{d e} / \rho_{d e}^{(0)}\right)^{1 / 2}
$$

remembering that $\Omega_{i}=\rho_{i} / \rho_{\text {crit }}$ for the $i-t h$ commponent, $\rho_{\text {crit }}$ is the critical density of the Universe and $\Omega_{i}^{(0)}=$ $\rho_{i}^{(0)} / \rho_{c r i t}^{(0)}=\left(8 \pi G / 3 H_{0}^{2}\right) \rho_{i}^{(0)}$. As an example, Figure 1 displays $w(z), \rho(z)$ and $H(z)$ for the first two harmonics of the Fourier series, the constant case and $\Lambda \mathrm{CDM}$.

\section{METHOLOGY}

In order to perform the parameter space exploration, and select the best-fit model, we make use of a modified version of a simple and fast Markov Chain Monte Carlo (MCMC) code that computes expansion rates and distances 

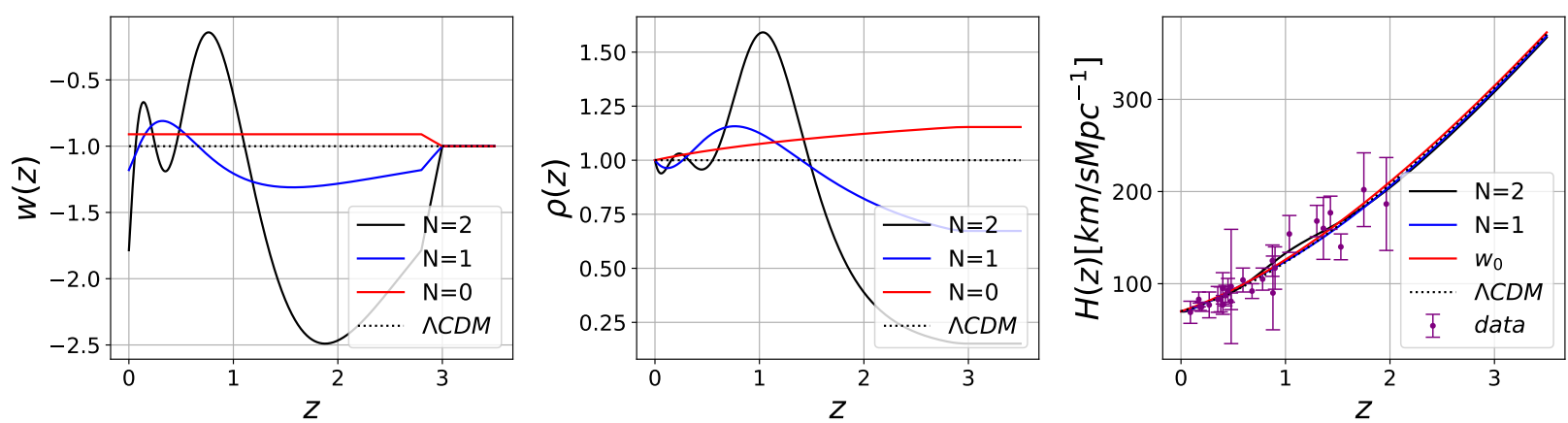

FIG. 1: Left panel: dark energy EoS parameter $w(z)$, middle panel: energy density $\rho(z)$ of the dark energy and right panel: Hubble function $H(z)$ with data from [31]. In solid black and blue lines the first two harmonics of the Fourier series $N=2,1$ (number of pairs of $a_{n}$ and $b_{n}$ of the expansion), in red the case with only the constant term $w_{0}$ of the series and the dotted black line corresponds to the $\Lambda \mathrm{CDM}$ model. The used values of $a_{n}, b_{n}$ and $w_{0}$ for this plot were taken from Table I.

from the Friedmann equation, named SimpleMC [29, 30]. The datasets considered throughout the analysis include a compressed version of the Planck data (PLK), a recent reanalysis of SNIa data, and high-precision BAO measurements at different redshifts up to $z<2.36$ [29]. We also include a collection of currently available $H(z)$ measurements (HD), see [31] and references therein. We assume a flat $\Lambda \mathrm{CDM}$ universe described by the following parameters: $\Omega_{b} h^{2}$ and $\Omega_{D M}$ are the physical baryon density and dark matter density, respectively, relative to the critical density, and $h$ the dimensionless Hubble parameter such that $H_{0}=100 \mathrm{hs}^{-1} \mathrm{Mpc}^{-1} \mathrm{~km}$. Here the neutrinos are massless and the effective number of relativistic species has the standard $\Lambda \mathrm{CDM}$ value of $N_{\mathrm{eff}}=3.04$. The code neglects perturbations for the dark energy, but this could be an important point to deal with in a future work. The SimpleMC code contains the Gelman-Rubin convergence criterion $(R)$, which typically is set up to be $0.97<R<1.03$; for an extended review of cosmological parameter inference in cosmology see [32]. In our Fourier description we introduce a set of free parameters $w_{0}, a_{n}, b_{n}$ to describe the overall shape of the dark energy equation-of-state $w(z)$. The transition points selected are $z_{i n i}=3.0$ and $z_{\text {med }}=2.8$. For each of them, we allow variations in amplitudes with conservative flat priors $w_{0}=[-3,-1]$ and $a_{n}, b_{n}=[-1.5,1.5]$. Left columns on Table I display the parameters used throughout each description of $w(z)$.

We perform a model comparison in order to select the best description for the equation-of-state $w(z)$. The main aim of model selection is to balance the goodness of fit to the observational data against the complexity of the model, in this case given by the extra free parameters. One way to carry out this process is by calculating the Bayesian evidence, which naturally incorporates a penalisation factor through the prior volume of the parameter space $[4,6]$. Calculating the evidence could be challenging and a computationally demanding process. In this work, however, for simplicity and noticing the near-gaussianity of the posterior distributions, we focus on the information criteria methods such as the Akaike Information Criterion (AIC). The AIC is defined as:

$$
\mathrm{AIC}=-2 \ln \mathcal{L}_{\max }+2 k,
$$

where the first term incorporates the goodness-of-fit through the likelihood $\mathcal{L}$, and the second term is interpreted as the penalisation factor given by two times the extra number of parameters $(k)$ of the model. The more complex the model is, the faster the penalty term takes over. For a small number of datapoints $N$, it is important to attach a correction term to the AIC [33], given by

$$
\mathrm{AIC}_{\mathrm{C}}=\mathrm{AIC}+\frac{2 k(k+1)}{N-k-1} .
$$

Therefore the preferred model is the one that minimises the $\mathrm{AIC}_{\mathrm{C}}$.

\section{RESULTS}

Table I displays the mean and $1 \sigma$ error values obtained during the analysis for the coefficients on each Fourier expansion. For each model the first row uses data from SNIa+BAO+HD, while the second row additionally includes 
TABLE I: Constraints on the set of parameters used on each description for $w(z)$. For one-tailed distributions the upper limit $95 \%$ C.L. is given and for two-tailed the $68 \%$ is shown. Its corresponding plot is displayed in Figure 3. For each parameterization of $w(z)$ we have considered two sets of data: first row contains SNIa+BAO+HD, while the second row additionally includes PLK data. Second column describes the specific model chosen in the text, with $x=\frac{2 \pi}{T}\left(\frac{1}{z+1}-\frac{1}{z_{m e d+1}}\right)$.

\begin{tabular}{lccccccccc}
\hline & Model & $w_{0}$ & $a_{1}$ & $b_{1}$ & $a_{2}$ & $b_{2}$ & $-2 \ln \mathcal{L}_{\max } \mathrm{S} / \mathrm{N}^{*} \Delta \mathrm{AIC}_{C}$ \\
\hline & $\Lambda$ CDM & -2 & 0 & 0 & 0 & 0 & 68.98 & 0 & 0 \\
& & -2 & 0 & 0 & 0 & 0 & 73.44 & 0 & 0 \\
\hline (a) & $\frac{w_{0}}{2}$ & $-1.82 \pm 0.12$ & 0 & 0 & 0 & 0 & 67.06 & $1.4 \sigma$ & 0.3 \\
& $-1.93 \pm 0.08$ & 0 & 0 & 0 & 0 & 73.84 & $0.5 \sigma$ & 2.6 \\
\hline (b) & $w_{0}+\frac{z}{1+z} a_{1}{ }^{* *}$ & $-0.87 \pm 0.11-0.28 \pm 0.58$ & 0 & 0 & 0 & 67.08 & $1.4 \sigma$ & 2.7 \\
& & $-0.94 \pm 0.10-0.11 \pm 0.34$ & 0 & 0 & 0 & 73.66 & $0.5 \sigma$ & 4.8 \\
\hline (c) & $\frac{w_{0}}{2}+a_{1} \sin (x)$ & $-2.06 \pm 0.32-0.16 \pm 0.20$ & 0 & 0 & 0 & 66.74 & $1.5 \sigma$ & 2.3 \\
& & $-2.01 \pm 0.12-0.10 \pm 0.11$ & 0 & 0 & 0 & 73.24 & $0.4 \sigma$ & 4.4 \\
\hline (d) & $\frac{w_{0}}{2}+b_{1} \cos (x)$ & $-1.78 \pm 0.13$ & 0 & $-0.11 \pm 0.12$ & 0 & 0 & 66.12 & $1.7 \sigma$ & 1.7 \\
& & $-1.94 \pm 0.08$ & 0 & $-0.06 \pm 0.11$ & 0 & 0 & 73.50 & $0.2 \sigma$ & 4.6 \\
\hline (e) & $\frac{w_{0}}{2}+a_{1} \sin (x)$ & $-2.12 \pm 0.33-0.22 \pm 0.21-0.12 \pm 0.13$ & 0 & 0 & 65.84 & $1.8 \sigma$ & 3.8 \\
& $+b_{1} \cos (x)$ & $-2.09 \pm 0.14-0.19 \pm 0.13-0.17 \pm 0.12$ & 0 & 0 & 71.56 & $1.4 \sigma$ & 5.1 \\
\hline (f) & $a_{1} \sin (x)+b_{1} \cos (x)$ & -2 & $-0.09 \pm 0.08$ & $-0.54 \pm 0.23-0.54 \pm 0.27$ & 0 & 61.52 & $2.7 \sigma$ & -0.5 \\
& $+a_{2} \sin (2 x)$ & -2 & $-0.07 \pm 0.09-0.26 \pm 0.12-0.27 \pm 0.17$ & 0 & 69.66 & $1.9 \sigma$ & 3.2 \\
\hline (g) $\frac{w_{0}}{2}+a_{1} \sin (x)+b_{1} \cos (x)$ & $-2.34 \pm 0.31-0.29 \pm 0.20-0.70 \pm 0.29-0.72 \pm 0.34$ & 0 & 61.24 & $2.8 \sigma$ & 1.7 \\
& $+a_{2} \sin (2 x)$ & $-2.53 \pm 0.24-0.38 \pm 0.17$ & $-0.70 \pm 0.23-0.76 \pm 0.28$ & 0 & 63.94 & $3.1 \sigma$ & -0.1 \\
\hline (h) $\frac{w_{0}}{2}+a_{1} \sin (x)+b_{1} \cos (x)$ & $-2.31 \pm 0.31-0.28 \pm 0.22-0.65 \pm 0.30-0.67 \pm 0.34$ & $0.02 \pm 0.23$ & 61.40 & $2.8 \sigma$ & 4.4 \\
& $+a_{2} \sin (2 x)+b_{2} \cos (2 x)$ & $-2.50 \pm 0.28-0.41 \pm 0.22$ & $-0.66 \pm 0.27-0.70 \pm 0.30$ & $0.13 \pm 0.26$ & 64.26 & $3.0 \sigma$ & 0.2 \\
\hline \hline
\end{tabular}

${ }^{*}$ Signal-to-noise ratio $=\sqrt{2 \Delta \ln \mathcal{L}}$ of $w(z)$ deviating from $\Lambda$ CDM based on the improvement in the fit alone.

${ }^{* *}$ This particular model corresponds to the CPL parameterization.

PLK data. The last three columns of the table contain statistical information in order to provide an insight for the best model. Notice that some of the best-fit values of the parameters are located right outside the $\Lambda$ CDM model, within statistical significance. Of particular interest are the last three models where deviations from the standard values $a_{1}=b_{1}=a_{2}=0$ are more noticeable, and hence leading to a significant improvement on the likelihood. For instance model (f), with three extra parameters and fixed $w_{0}$, has deviations from $\Lambda$ CDM at about $2 \sigma$-according to the signal-to-noise ratio in the fit alone-- Moreover, if $w_{0}$ is let to be a free parameter in model $(\mathrm{g})$, the best-fit improves significantly as a consequence of $w_{0}$ being different from the standard value $w_{0}=-2$ and deviations from $\Lambda$ CDM increment up to $3 \sigma$. The inclusion of extra parameters improves the fit to the data (dash lines in Figure 2), however, also carries out a penalisation factor that affects directly the Akaike criteria (solid lines in Figure 2). That is, even though model (h) contains an extra parameter $b_{2}$, however, it has no impact on improving the fit considerably. This is a consequence of $b_{2}$ being close to zero and hence providing no contribution to enhance the description of the data. Nevertheless, because of this increment of the number of parameters the penalty term takes over and hence the AIC value raises back again. We can go even further adding parameters, however as we have seen, the penalisation factor dominates and also more freedom brings more correlations between parameters and therefore noisier reconstructions. Figure 3 shows the posterior distribution (with $1 \sigma$ and $2 \sigma$ confidence levels) for the equation-of-state $w(z)$ given the set of MC chains for each description. As expected, adding parameters provides more structure than the cosmological constant. Let us have a look again at the last three models, where the shape of $w(z)$ resembles a similar form already obtained in previous analyses, i.e. [4-6]. Throughout these reconstructions we notice the presence of two peaks, the major one located at $z \sim 0.8$ and a small one at $z \sim 0.2$ (similar positions to the ones obtained in paper [5]). It is also observed that at the present time $(z=0)$ and high redshifts $(z>1)$ slightly favoured $w<-1$, while at redshift $(z \sim 0.8) w>-1$ is preferred, and hence the reconstructed $w(z)$ exhibits the crossing of the PDL several times. The crossing of the PDL plays a key role in identifying the correct dark energy model. If future surveys confirm its existence, single scalar field theories (with minimal assumptions) might be in serious problems as they cannot reproduce this essential feature, and therefore alternative models should be considered. A key point to stress out is that the cosmological constant $w=-1$ lays down far outside of the $2 \sigma$ region (outer solid black line), particularly at high redshift on the second plot of model (g) of Figure 3. The richness of this form is a consequence of releasing some 


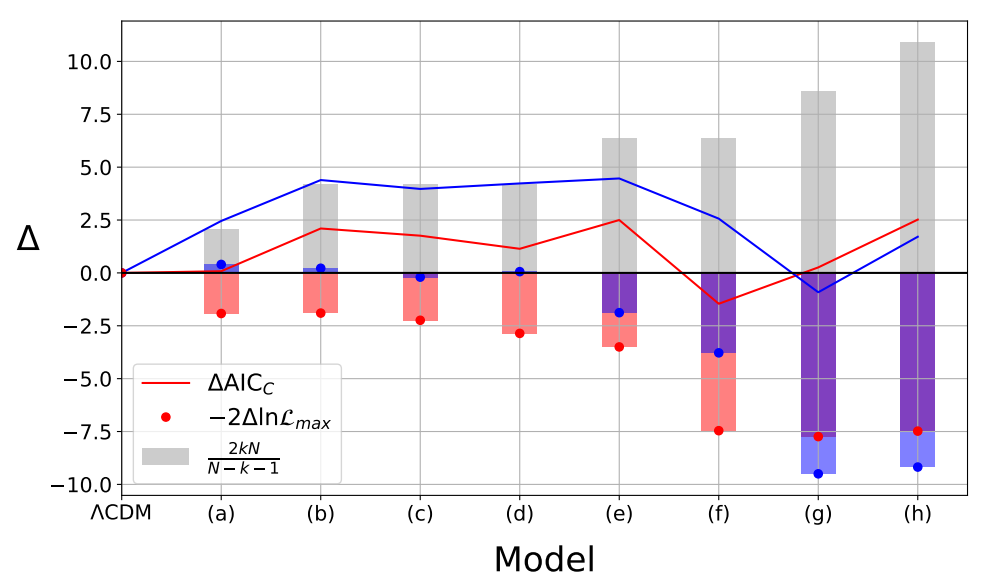

FIG. 2: Red bar plots show the inclusion of extra parameters improves the fit to the data, seen through $-2 \Delta \ln \mathcal{L}_{\max }$ (compare to $\Lambda \mathrm{CDM}$ ). We can go even further adding parameters, however this increment causes that penalty term (gray bar plot) dominates and hence the $\mathrm{AIC}_{\mathrm{C}}$ value raises back again. Therefore, the preferred model is the one that minimises the $\mathrm{AIC}_{\mathrm{C}}$ (solid lines). Blue colour additionally includes PLK data.

tensions between datasets, specially for the high- $z$ BAO.

Of all the models presented in Table I, model (g) deviates the most from the cosmological constant $(3.1 \sigma)$. Figure 4 displays $1 \mathrm{D}$ and $2 \mathrm{D}$ marginalised posterior distributions for the parameters used to describe model (g). The vertical dashed lines, which correspond to the $\Lambda \mathrm{CDM}$ model, give an insight to the amount of deviation that each parameter presents, in particular parameters $b_{1}$ and $a_{2}$ deviate the most from the standard values. Here we also notice some parameters are highly correlated. In a future work we would perform a dimensional reduction analysis (i.e. PCA) which decreases the penalty factor, but still preserves a similar shape of $w(z)$ and hence $-2 \ln \mathcal{L}$ keeps a similar value. On the other hand, the right panel of Figure 4 displays the $w(z)$ and $\rho(z)$ contributions from each term in the Fourier expansion. The $a_{2}$ parameter contribution looks very alike to the full Fourier expansion, and hence its importance in the reconstruction. Similarly the $b_{1}$ parameter enhances the amplitude of the major peak and provides contributions to the low-negative values of $w(z)$ and redshift today.

\section{CONCLUSIONS AND DISCUSSIONS}

In this work were proposed Fourier series to describe the dynamical dark energy EoS parameter $w(z)$. This approach reproduces the oscillating behaviour of $w(z)$ shown in reconstructions from observational data. Also generalises previous dynamical dark energy proposals from trigonometrical functions and avoids some problems inherent of models based on the Taylor series, like the divergence at relative high redshift $(\sim 3)$. For several selected cases of the Fourier series, the parameters were constrained from data using a simpleMC and compared with other models such as $\Lambda \mathrm{CDM}$ and a particular case of Taylor series (CPL model). We noticed that as the number of parameters increase (i.e. terms of the series), more correlations amongst them are created, and also an increment to the penalisation factor in the Akaike criteria. The Fourier series approach yields to a better fit to the data by more than $3 \sigma$ in comparison to the cosmological constant $w=-1$, therefore having a clear preference for a dynamical dark energy behaviour. Moreover, if we compare the particular model $(\mathrm{g})$ against the CPL parameterization, we found that model $(\mathrm{g})$ is preferred by more than $3 \sigma$, also the difference in the Akaike criteria is $\Delta \mathrm{AIC}_{\mathrm{C}}=-4.9$, which can be considered as a strong evidence against the CPL description. With this analysis, in a model independent way, we are able to discriminate the cosmological constant and provide a better fit than the Taylor expansion - in particular the CPL parameterization-. We have considered the $\mathrm{AIC}_{\mathrm{C}}$ to penalise the extra parameters introduced in our analysis. Even though this penalisation factor acts strongly, the criteria still tells us that a Fourier expansion provides a slightly better explanation of the data, specially to the inclusion of BAO at high redshift. This first analysis of considering $w(z)$ as a Fourier series, has its success in having a natural oscillatory behaviour and being compatible with model-independent reconstructions. More and better observational data are needed to test in more detail this proposal, however, it seems that the multiple crosses of the PDL are unavoidable, putting in conflict some simple models for dark energy such as the cosmological constant, simple scalar fields, CPL and low-order Taylor series. 

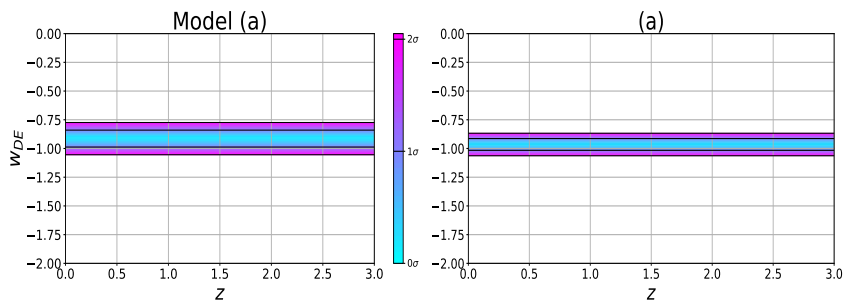

(c)
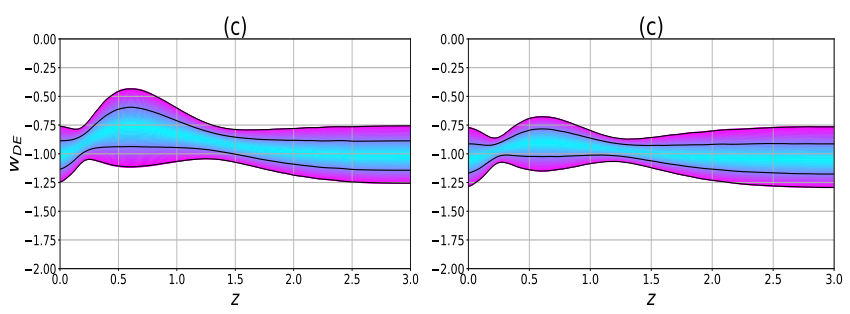

(e)
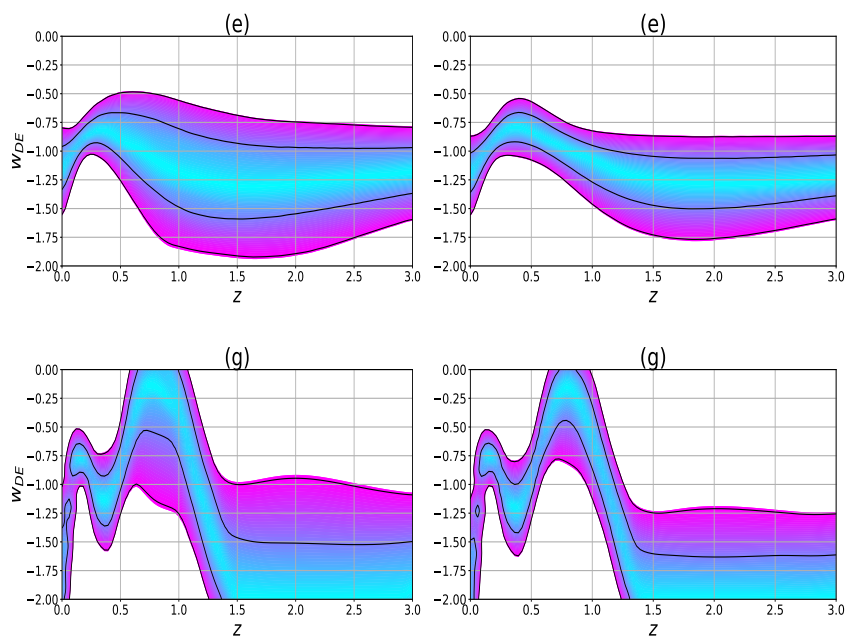
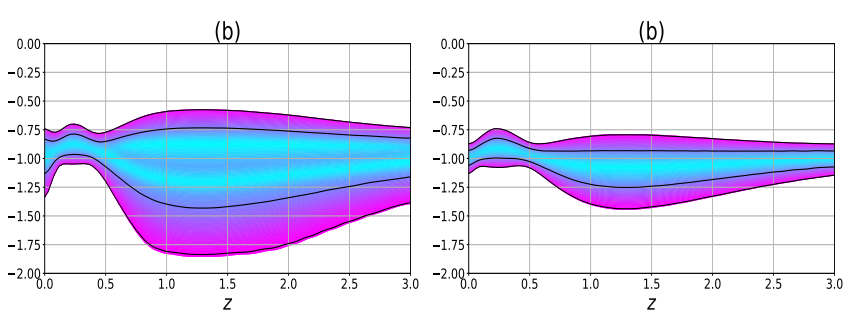

(d)
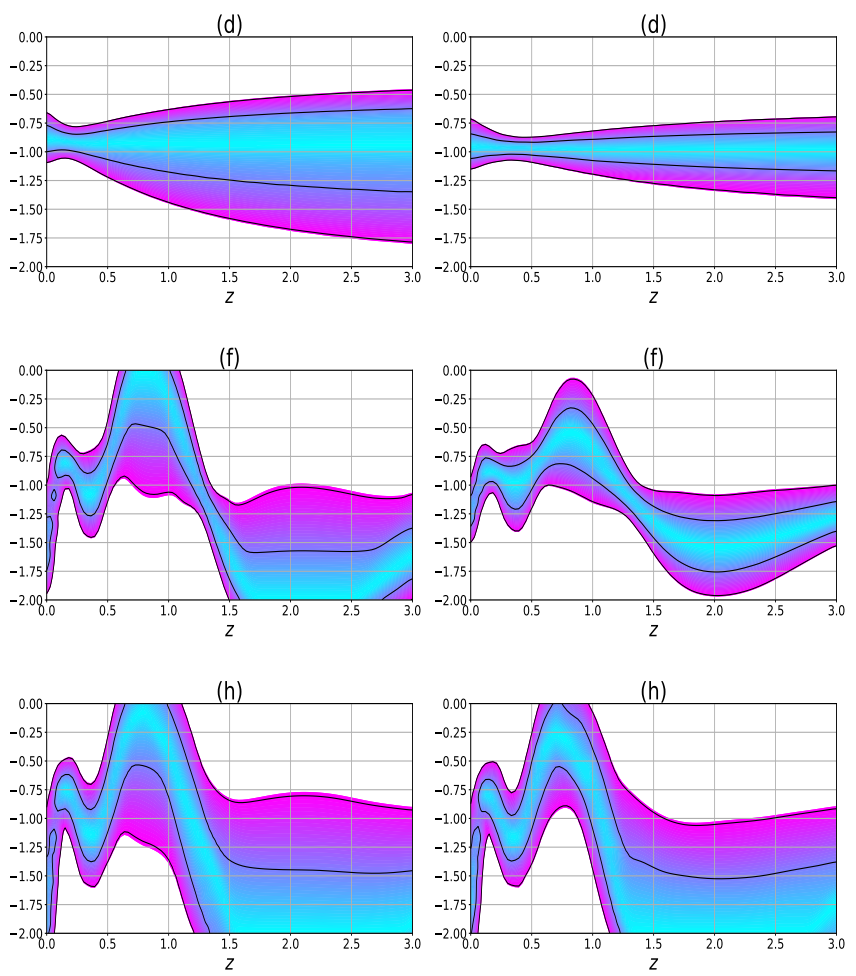

FIG. 3: These plots show the posterior probability $\operatorname{Pr}(w \mid z)$ : the probability of $w$ as normalised in each slice of constant $z$, with colour scale in confidence interval values. The $1 \sigma$ and $2 \sigma$ confidence intervals are plotted as black lines. Left panel for each set contains $\mathrm{SN}+\mathrm{BAO}+\mathrm{HD}$ datasets, while the right panel includes additionally PLK data.

\section{ACKNOWLEDGEMENTS}

JAV acknowledges the support provided by FOSEC SEP-CONACYT Investigación Básica A1-S-21925, and DGAPA-PAPIIT IA102219. DT acknowledge financial support from CONACYT Mexico postdoctoral fellowships. The authors thank Josué De-Santiago for the revision of the manuscript.

[1] P. A. R. Ade et al. [Planck Collaboration], Astron. Astrophys. 594 (2016) A13 doi:10.1051/0004-6361/201525830 [arXiv:1502.01589 [astro-ph.CO]].

[2] A. G. Riess et al., Astrophys. J. 826 (2016) no.1, 56 doi:10.3847/0004-637X/826/1/56 [arXiv:1604.01424 [astro-ph.CO]].

[3] T. Delubac et al. [BOSS Collaboration], Astron. Astrophys. 574 (2015) A59 doi:10.1051/0004-6361/201423969 [arXiv:1404.1801 [astro-ph.CO]].

[4] J. Alberto Vazquez, M. Bridges, M. P. Hobson and A. N. Lasenby, JCAP 1209 (2012) 020 doi:10.1088/14757516/2012/09/020 [arXiv:1205.0847 [astro-ph.CO]].

[5] G. B. Zhao et al., Nat. Astron. 1 (2017) no.9, 627 doi:10.1038/s41550-017-0216-z [arXiv:1701.08165 [astro-ph.CO]].

[6] S. Hee, J. A. Vázquez, W. J. Handley, M. P. Hobson and A. N. Lasenby, Mon. Not. Roy. Astron. Soc. 466 (2017) no.1, 369 doi:10.1093/mnras/stw3102 [arXiv:1607.00270 [astro-ph.CO]]. 

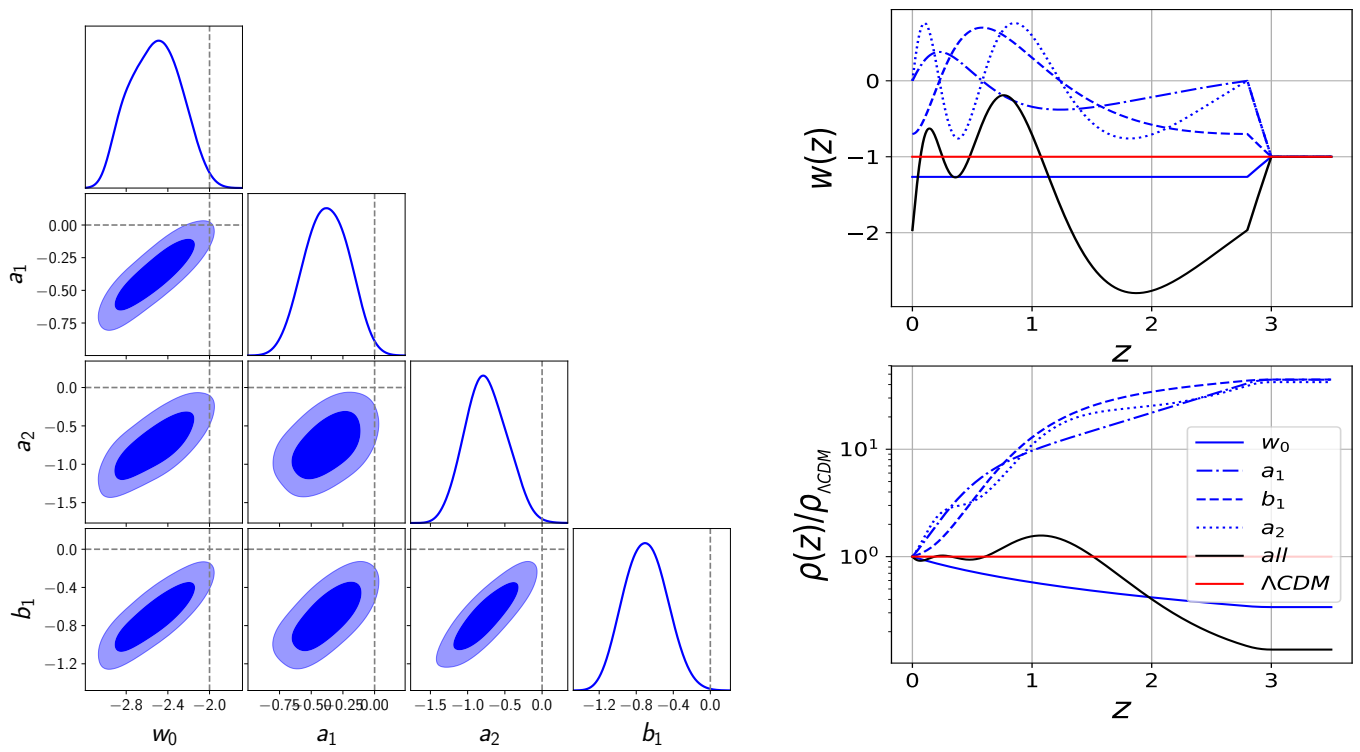

FIG. 4: Left panel: 1D and 2D probability posterior distributions of the parameters used in model (g) with the incorporation of PLK data. Vertical dashed lines correspond to the $\Lambda$ CDM values. Right panel: $w(z)$ and $\rho(z)$ contributions from each term in the Fourier expansion. The best-fit values of the left panel were used in this plot.

[7] Y. Wang, L. Pogosian, G. B. Zhao and A. Zucca, Astrophys. J. 869 (2018) L8 doi:10.3847/2041-8213/aaf238 [arXiv:1807.03772 [astro-ph.CO]].

[8] H. E. S. Velten, R. F. vom Marttens and W. Zimdahl, Eur. Phys. J. C 74 (2014) no.11, 3160 doi:10.1140/epjc/s10052014-3160-4 [arXiv:1410.2509 [astro-ph.CO]].

[9] A. Padilla, arXiv:1502.05296 [hep-th].

[10] M. López-Corredoira, Found. Phys. 47 (2017) no.6, 711 doi:10.1007/s10701-017-0073-8 [arXiv:1701.08720 [astro-ph.CO]].

[11] Arun K., Gudennavar S., Sivaram C., Advances in Space Research, (2017), 60, 166 doi:10.1016/j.asr.2017.03.043

[12] T. Clifton, P. G. Ferreira, A. Padilla and C. Skordis, Phys. Rept. 513 (2012) 1 doi:10.1016/j.physrep.2012.01.001 [arXiv:1106.2476 [astro-ph.CO]].

[13] W. Zimdahl, AIP Conf. Proc. 1471 (2012) 51 doi:10.1063/1.4756811 [arXiv:1204.5892 [astro-ph.CO]].

[14] O. Akarsu, T. Dereli and J. A. Vazquez, JCAP 1506 (2015) no.06, 049 doi:10.1088/1475-7516/2015/06/049 [arXiv:1501.07598 [astro-ph.CO]].

[15] Yoo J., Watanabe Y., Int. J. Mod. Phys., D21, (2012), 1230002

[16] M. Chevallier and D. Polarski, Int. J. Mod. Phys. D 10 (2001) 213 doi:10.1142/S0218271801000822 [gr-qc/0009008].

[17] E. V. Linder, Phys. Rev. Lett. 90 (2003) 091301 doi:10.1103/PhysRevLett.90.091301 [astro-ph/0208512].

[18] S. Kumar and L. Xu, Phys. Lett. B 737 (2014) 244 doi:10.1016/j.physletb.2014.08.059 [arXiv:1207.5582 [gr-qc]].

[19] R. J. Scherrer, Phys. Rev. D 92 (2015) no.4, 043001 doi:10.1103/PhysRevD.92.043001 [arXiv:1505.05781 [astro-ph.CO]].

[20] B. Sagredo, J. S. Lafaurie and D. Sapone, arXiv:1808.05660 [astro-ph.CO].

[21] Dai J.-P., Yang Y., Xia J.-Q., The Astrophysical Journal 857, (2018), 9 doi:10.3847/1538-4357/aab49a

[22] C. J. A. P. Martins and M. P. Colomer, Astron. Astrophys. 616 (2018) A32 doi:10.1051/0004-6361/201833313 [arXiv:1806.07653 [astro-ph.CO]].

[23] E. V. Linder, Astropart. Phys. 25 (2006) 167 doi:10.1016/j.astropartphys.2005.12.003 [astro-ph/0511415].

[24] Pace F., Fedeli C., Moscardini L., Bartelmann M., Mon. Not. Roy. Astron. Soc., 422, (2012), 1186 doi:10.1111/j.13652966.2012.20692.x

[25] A. Kurek, O. Hrycyna and M. Szydlowski, Phys. Lett. B 659 (2008) 14 doi:10.1016/j.physletb.2007.10.074 [arXiv:0707.0292 [astro-ph]].

[26] S. Pan, E. N. Saridakis and W. Yang, Phys. Rev. D 98 (2018) no.6, 063510 doi:10.1103/PhysRevD.98.063510 [arXiv:1712.05746 [astro-ph.CO]].

[27] G. Panotopoulos and . Rincn, Phys. Rev. D 97 (2018) no.10, 103509 doi:10.1103/PhysRevD.97.103509 [arXiv:1804.11208 [astro-ph.CO]].

[28] L. G. Jaime, M. Jaber and C. Escamilla-Rivera, Phys. Rev. D 98 (2018) no.8, 083530 doi:10.1103/PhysRevD.98.083530 [arXiv:1804.04284 [astro-ph.CO]].

[29] É. Aubourg et al., Phys. Rev. D 92 (2015) no.12, 123516 doi:10.1103/PhysRevD.92.123516 [arXiv:1411.1074 [astro-ph.CO]].

[30] Slosar, https://github.com/slosar/april, (2015) 
[31] A. Gómez-Valent and L. Amendola, JCAP 1804 (2018) no.04, 051 doi:10.1088/1475-7516/2018/04/051 [arXiv:1802.01505 [astro-ph.CO]].

[32] L. E. Padilla, L. O. Tellez, L. A. Escamilla and J. A. Vazquez, arXiv:1903.11127 [astro-ph.CO].

[33] Burnham K. P., Anderson D. R., Model selection and multimodel inference: a practical information-theoretic approach, 2 edn. Springer (2002) 